\title{
Closing the Brief Case: Too Beta To Be a "B"
}

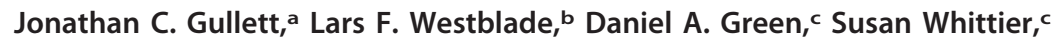 \\ Eileen M. Burda,d,e \\ Department of Pathology and Laboratory Medicine, Emory University School of Medicine, Atlanta, Georgia, \\ USAa; Department of Pathology and Laboratory Medicine, Weill Cornell Medical College, New York, New \\ York, USA ${ }^{\text {b; }}$ Department of Pathology and Cell Biology, Columbia University Medical Center, New York, \\ New York, USAc; Department of Medicine, Division of Infectious Diseases, Emory University School of \\ Medicine, Atlanta, Georgia, USAd; Emory Antibiotic Resistance Center, Emory University School of \\ Medicine, Atlanta, Georgia, USAe
}

KEYWORDS Streptococcus pseudoporcinus, adverse obstetrical outcomes, betahemolytic streptococci, group B streptococci

\section{ANSWERS TO SELF-ASSESSMENT QUESTIONS}

1. Which of the following characteristics suggests that an isolate of Gram-positive cocci in short chains that is catalase negative and Lancefield group B antigen positive may be Streptococcus pseudoporcinus?

A. A narrow zone of beta-hemolysis, esculin hydrolysis negativity, and hippurate positivity

B. A narrow zone of beta-hemolysis, esculin hydrolysis positivity, and hippurate positivity

C. A wide zone of beta-hemolysis, esculin hydrolysis positivity, and hippurate negativity

D. A wide zone of beta-hemolysis, esculin hydrolysis negativity, and hippurate positivity

Answer: $C$. Isolates that react with Lancefield group B antigen agglutination reagents and demonstrate a wide zone of beta-hemolysis should raise suspicion for $S$. pseudoporcinus. S. pseudoporcinus is esculin hydrolysis positive and hippurate negative. A CAMP test, if performed, is positive. Pyrrolidonyl arylamidase reactivity is varied and can be either positive or negative, depending on the strain.

2. What is the normal habitat for Streptococcus pseudoporcinus in humans?
A. Vagina
B. Skin
C. Mouth
D. Conjunctiva

Answer: A. S. pseudoporcinus inhabits the genitourinary tracts of some women and has been recovered from 1.0 to $5.4 \%$ of vaginal-rectal specimens submitted for the detection of S. agalactiae.

3. Streptococcus pseudoporcinus is possibly associated with which of the following adverse obstetrical outcomes?
A. Neural tube defects
B. Neonatal sepsis
C. Pregnancy-induced hypertension
D. Premature rupture of fetal membranes and cervical insufficiency

Citation Gullett JC, Westblade LF, Green DA Whittier S, Burd EM. 2017. Closing the Brief Case: Too beta to be a "B." J Clin Microbiol 55:1973-1974. https://doi.org/10.1128/JCM .02390-16.

Editor Carey-Ann D. Burnham, Washington University School of Medicine

Copyright $\odot 2017$ American Society for Microbiology. All Rights Reserved. Address correspondence to Eileen M. Burd, eburd@emory.edu.

See page 1604 in this issue (https://doi.org/10 $.1128 / J C M .02387-16)$ for case presentation and discussion. 
Answer: D. Although there is not a definitive association, reports in the literature point to a possible connection between S. pseudoporcinus and premature delivery due to rupture of fetal membranes as well as cervical insufficiency.

\section{TAKE-HOME POINTS}

- Streptococcus pseudoporcinus can be recovered from vaginal-rectal specimens submitted for the detection of Streptococcus agalactiae.

- Although S. pseudoporcinus belongs to Lancefield antigen groups NG1, E, and $\mathrm{P}$ or may even be untypeable, isolates of S. pseudoporcinus often cross-react with Lancefield group $B$ antigen agglutination reagents.

- Colonies of both S. pseudoporcinus and S. agalactiae are large (>0.5 mm) after $24 \mathrm{~h}$ of incubation, but S. pseudoporcinus has a wide zone of beta-hemolysis on sheep blood agar after overnight incubation compared to the narrow zone (slightly bigger than the colony formed) of subtle beta-hemolysis that is produced by most strains of S. agalactiae.

- Risk factors for vaginal-rectal colonization include black ethnic groups, recent Trichomonas vaginalis infection, primary or recurrent genital herpes simplex virus infection, bacterial vaginosis, obesity, and two or more male sexual partners during the 1 or 2 months since the last health clinic visit (i.e., increased numbers of sexual partners).

- Vaginal-rectal colonization may be associated with complications of pregnancy, including preterm rupture of fetal membranes and cervical insufficiency. 